\title{
Donor smoking reduces recipient survival after lung transplant
}

This study investigated whether lung transplantation from donors with a positive smoking history was associated with increased morbidity and mortality in recipients. Data were analysed from 2181 lung transplant waiting-list patients in whom 1295 lung transplants from brain-dead donors were carried out in the UK between 1999 and 2010. Sequential stratification was used to assess survival from waiting list registration in those receiving lung transplants from donors with positive smoking histories as compared with matched controls on the waiting list not receiving such transplants. Primary outcome was survival post transplant at 3 years and secondary outcomes included recipients' cause of death, post-transplant hospital and intensive therapy unit (ITU) length of stay and $\mathrm{FEV}_{1}$ at 2 years in those receiving bilateral transplants.

Recipients of lungs transplanted from donors with a positive smoking history had lower survival (median 4.9 years vs 6.5 years), longer hospital/ITU stays and a $10 \%$ reduced $\mathrm{FEV}_{1}$ at 2 years compared with those receiving lungs from non-smokers. All cause survival was inferior at 30 days and rates of death due to graft failure were increased after 90 days in patients receiving lungs from donors with a positive smoking history. Lungs from donors with predicted cigarette consumption $>20$ /day were associated with the worst recipient outcomes.

Thirty-nine per cent of transplants were from donors with a positive smoking history. Although transplantation of such lungs is associated with increased morbidity and mortality compared with those from non-smokers, overall waiting list mortality is reduced by their acceptance (adjusted HR for death at 3 years 1.36). Recipients with the greatest benefit were those with septic and fibrotic lung diseases. Whether a pack-year threshold above which lungs from donors who smoke should be rejected is yet to be established but caution is advised in those with greater than 20 pack years.

- Bonser RS, Taylor R, Collettt D, et al. Effect of donor smoking on survival after lung transplantation: a cohort study of a prospective registry. Lancet 2012;380:747-55.

\section{Robert Derrick}

Correspondence to Dr Robert Derrick, FY2, Department of Emergency Medicine, Princess Royal Hospital, Farnborough Common, Orpington, Kent BR6 8ND, UK; robert.derrick@nhs.net

Competing interests None.

Provenance and peer review Not commissioned; externally peer reviewed.

To cite Derrick R. Thorax 2013;68:921.

Published Online First 1 November 2012

Thorax 2013;68:921. doi:10.1136/thoraxjn-2012-202849 